\title{
Mean-Dispersion Preferences with a Specific Dispersion Function
}

\author{
Mark Schneider* and Manuel Nunez ${ }^{\dagger}$
}

May 25, 2016

\begin{abstract}
A popular approach to modeling ambiguity aversion is to decompose preferences into the subjective expected utility of an act and an ambiguity index, or an adjustment factor, or a dispersion function. However, in these approaches the dispersion function (or ambiguity index, or adjustment factor) has very little structure imposed on it, leaving the selection of a specific dispersion function in applications to be rather arbitrary. In this note, working in the AnscombeAumann (1963) framework, we provide a simpler axiomatic characterization of mean-dispersion preferences which uniquely identifies the dispersion function from the infinite class of possible alternatives. Given the representation, we also obtain unique identification of subjective probabilities.

JEL classification: D81.

Keywords: ambiguity aversion, translation invariance, dispersion, uncertainty, probabilistic sophistication.
\end{abstract}

\section{Introduction}

One popular approach to modeling attitudes toward ambiguity is to decompose preferences into the expected utility of an act and an ambiguity index (Maccheroni et al., 2006a,b), or an adjustment factor (Siniscalchi, 2009),

\footnotetext{
*Economic Science Institute, Chapman University, One University Drive, Orange, CA 92866 E-mail: Schneide@chapman.edu.

${ }^{\dagger}$ School of Business, University of Connecticut, 2100 Hillside Road Unit 1041, Storrs, CT 06269-1041. E-mail: mnunez@business.uconn.edu.
} 
or a dispersion function (Grant and Polak, 2013). The most general of these specifications is the class of mean-dispersion preferences, axiomatized in the Anscombe-Aumann (1963) framework by Grant and Polak (2013). The mean-dispersion class is quite large and includes the multiple priors model (Gilboa and Schmeidler, 1989), Choquet expected utility (Schmeidler, 1989), invariant bi-separable preferences (Ghirardato et al., 2004), the variational representation of preferences (Maccheroni et al., 2006a,b), and vector expected utility (Siniscalchi, 2009), as special cases. Mean-dispersion preferences, as characterized by Grant and Polak (2013) can be represented as:

$$
V(f, \pi)=\mu(f, \pi)-\rho(d(f, \pi)),
$$

where $\mu(f, \pi)$ is the mean utility of act $f$ with respect to a vector probability distribution $\pi$ across all states of nature; and $d(f, \pi)$ is the vector of deviations from the mean utility (i.e, $d_{s}:=\mu(f, \pi)-U(f(s))$, where $U(f(s))$ is the expected utility of $f$ in state $s)$. The function $\rho(\cdot)$ is a measure of (aversion to) dispersion. While the generality of a representation theorem is very desirable, Grant and Polak (2013) comment that their main theorem is "too general to be very useful" (p. 1367). In particular, "any probability $\pi$ in $\Delta(S)$ is associated with a mean-dispersion representation of the preferences" (Grant and Polak, 2013, p. 1367). In addition, the dispersion function in the Grant-Polak representation, like the ambiguity index for variational preferences and the adjustment factor in vector expected utility has very little structure imposed on it ${ }^{1}$. Grant and Polak (2013) remark, "Typically, we will be interested in mean-dispersion preferences that at least partially tie down the admissible probabilities and that put more structure on the dispersion functions" (p. 1367). Indeed, even if one found that the axioms of variational preferences, or vector expected utility preferences, or mean-dispersion preferences are satisfied, one would be at a loss to determine which dispersion measure to use in applications, and the choice of any particular dispersion measure would be rather arbitrary.

In this note, working in the Anscombe-Aumann framework, we provide a simpler axiomatic characterization of mean-dispersion preferences, which uniquely selects the dispersion function from an infinite class of possible alternatives and which uniquely determines the subjective probability distribution

\footnotetext{
${ }^{1}$ Siniscalchi (2009) does provide a sharp representation of vector expected utility preferences in which the adjustment factor is pinned down up to rotations.
} 
over states. In particular, we characterize preferences of the form:

$$
f \succ g \text { iff } V(f, \pi)>V(g, \pi)
$$

where

$$
V(f, \pi):=\mu(f, \pi)-\rho r(f, \pi)
$$

and

$$
r(f, \pi):=\frac{1}{2} \sum_{s \in S} \pi_{s}|\mu(f, \pi)-U(f(s))|,
$$

where $\rho$ is a scalar in $[-1,1]$ and represents a continuous index that captures the decision maker's attitude toward utility dispersion across states, ranging from very dispersion-seeking $(\rho=-1)$ to very dispersion averse $(\rho=1)$. Alternatively, $\rho$ may be interpreted as an index of ambiguity attitude, and this interpretation is supported by our analysis in Section 5. As we establish at the end of Section 3, if the representation (1) is unbounded and $\rho$ is in $[0,1]$, then $\pi$ is unique and the preferences in (1) also admit a vector expected utility representation, an invariant bi-separable representation, and a multiple priors representation.

To obtain the representation in (1), we introduce the notion of perturbed lotteries to account for a decision maker's aversion to the risk that the true state is undesirable. Using the perturbed lotteries, well-established axioms in the literature (see Axioms 1 through 6 below), and one new axiom relating preferences among regular acts to preferences among perturbed acts (Axiom 7), we are able to obtain representation (1).

A somewhat different mean-dispersion model has been developed in a companion paper (Schneider and Nunez, 2015), in which a parallel approach to that of Klibanoff et al. (2005) is followed through the use of second order acts. In addition, the paper in Schneider and Nunez (2015) explicitly assumes the existence of the coefficient in the dispersion function. There is some concern in the literature that the use of second-order acts is problematic since second-order acts may not be observable. Al-Najjar and De Castro (2010) formalize this concern and show that there is "no verification mechanism to determine what the decision maker receives under a second-order act ... even in idealized repeated experiments where infinite data can be observed". As such, there has been considerable work aimed at axiomatizing a smooth ambiguity aversion model as in Klibanoff et al. (2005) but without relying on second-order acts. Other axiomatic characterizations of second order expected utility have been provided in many alternative setups including Nau 
(2006), Ergin and Gul (2009), Grant et al. (2009), Seo (2009), and Neilson (2010). For the same reason, it is desirable to axiomatize a model of mean-dispersion preferences that uniquely identifies the dispersion function without invoking second-order acts.

In this note, we show that it is possible to axiomatize a model of meandispersion preferences which uniquely derives the dispersion function and the coefficient representing attitudes toward dispersion, and which uniquely determines the subjective prior over states in the standard Anscombe-Aumann setting. This approach also makes the proof more involved than in the paper by Schneider and Nunez (2015). Moreover, we arrive at our representation using standard axioms in the literature plus one new axiom. We thereby provide a model which uniquely characterizes the dispersion function, in contrast to more general approaches in Maccheroni et al. (2006a,b), Siniscalchi (2009), and Grant and Polak (2013), which admit an infinite set of possible dispersion measures and so are difficult to specify in applications.

Sections 2 and 3 develop an axiomatic foundation for preferences in (1), where the absolute deviation dispersion function $r(f, \pi)$, the existence, uniqueness, and range of $\rho$, and the uniqueness of $\pi$, can be derived from the axioms. Section 4 designs a simple behavioral test of our new axiom, "bounded ambiguity preference." We show that the model explains aversion to ambiguity in Ellsberg's paradoxes in Section 5. Section 6 concludes.

\section{Objective and Subjective Lotteries}

We work in the classical setup of Anscombe and Aumann (1963). Let $X$ denote a set of outcomes with at least two elements. An objective lottery, $p$ : $X \rightarrow[0,1]$, is a finite support probability distribution over outcomes. That is, $p(x)=0$ for all but a finite number of outcomes in $X$ and $\sum_{x \in X} p(x)=1$. We denote by $\Delta(X)$ the set of objective lotteries and assume that it is a mixture space. As usual, a von Neumann-Morgenstern utility function is an application $U: \Delta(X) \rightarrow \mathbb{R}$ defined as

$$
U(p):=\sum_{x \in X} p(x) u(x)
$$

where $u: X \rightarrow \mathbb{R}$ is a utility function on the outcomes set.

Let $S=\left\{s_{1}, \ldots, s_{n}\right\}$ be a finite nonempty set representing all possible states of nature. We define a subjective lottery or act $f$ as any mapping 
$f: S \rightarrow \Delta(X)$. We will use both $f(s)$ and $f_{s}$ to denote the value of $f$ at state $s$. Notice that $f_{s}(x) \in[0,1]$ for all $s \in S$ and $x \in X$, and $\sum_{x \in X} f_{s}(x)=1$ for all $s \in S$. We denote the set of subjective lotteries (acts) by $\mathcal{F}$ and assume that it is a mixture space. The set of probability vectors on $S$ is denoted by $\Delta(S)$. Given a utility function $u: X \rightarrow \mathbb{R}$ and $\pi \in \Delta(S)$, we denote by $\mu(f, \pi)$ the mean utility of the subjective lottery $f$ in $\mathcal{F}$. That is,

$$
\mu(f, \pi):=\sum_{s \in S} \pi_{s} U(f(s))=\sum_{s \in S} \sum_{x \in X} \pi_{s} f_{s}(x) u(x) .
$$

We denote by " $\succ$ " $\subset \mathcal{F} \times \mathcal{F}$ a binary relation over $\mathcal{F}$. The relation $\succ$ is called a preference relation if it is asymmetric and negatively transitive, and in that case, we say that $f$ is preferred to $g$ if $f \succ g$. We say that $f$ is weakly preferred to $g$, denoted as $f \succsim g$, if $g \nsucc f$. Moreover, we say that $f$ is indifferent to $g$, denoted as $f \sim g$, if $f \nsucc g$ and $g \nsucc f$. Observe that if $\succ$ is a preference relation, then for all $f$ and $g$ exactly one of $f \succ g, g \succ f$, or $f \sim g$ holds; and $\succsim$ is a complete and transitive relation (Kreps, 1988).

A constant act $f$ is a subjective lottery that yields the same objective lottery in each state of nature: $f(s)=p$ for all $s \in S$, where $p \in \Delta(X)$ is an objective lottery. In this case, and when the context is clear, we also let $p \in \Delta(X)$ denote the corresponding constant subjective lottery. Accordingly, we can naturally extend the preference relation to $\Delta(X)$ by letting $p \succ q$, for $p, q \in \Delta(X)$, whenever the constant act yielding lottery $p$ for all states is strictly preferred to the constant act yielding lottery $q$ for all states. Furthermore, a degenerate objective lottery that yields outcome $x \in X$ with probability 1 is, once again abusing notation, denoted by $x$. Hence, we denote $x \succ y$ for $x, y \in X$ when outcome $x$ is preferred to outcome $y$. Clearly, for a constant act $p, \mu(p, \pi)=U(p)$ for all $\pi$.

We state the following axioms, which will be used in establishing our main result:

Axiom 1 (Preference) $\succ$ on $\mathcal{F}$ is a preference relation.

Axiom 2 (Continuity) For every $f, g, h \in \mathcal{F}$, the sets $\{\alpha \in[0,1]: \alpha f+(1-$ $\alpha) g \succsim h\}$ and $\{\alpha \in[0,1]: h \succsim \alpha f+(1-\alpha) g\}$ are closed.

Axiom 3 (Constant act independence) $p \succ p^{\prime}$ in $\Delta(X)$ implies $\alpha p+(1-$ $\alpha) q \succ \alpha p^{\prime}+(1-\alpha) q$ for all $q \in \Delta(X)$ and $\alpha \in(0,1]$. 
Axiom 4 (Non-degeneracy) There exist $f$ and $g$ in $\mathcal{F}$ such that $f \succ g$.

Axioms 1, 2, and 4 are standard and well understood. Axiom 3 is a classical axiom in the context of objective lotteries and a weak version of the certainty independence axiom for acts used by Gilboa and Schmeidler (1989) and Ghirardato et al. (2004). The axioms together yield the classical von Neumann-Morgenstern representation theorem when restricted to the set $\Delta(X)$ of objective lotteries:

Theorem 1 If relation $\succ$ satisfies Axioms 1 through 4, then there exists a nonconstant affine function $u: X \rightarrow \mathbb{R}$ such that

$$
p \succ q \text { if and only if } U(p)>U(q)
$$

for all $p, q \in \Delta(X)$.

\section{Perturbed Lotteries and Mean-Dispersion Preferences}

Given $\pi \in \Delta(S)$ and $f \in \mathcal{F}$, we let $f_{\pi}$ denote the objective lottery obtained from averaging all the objective lotteries in act $f$. That is,

$$
f_{\pi}:=\sum_{s \in S} \pi_{s} f(s) .
$$

Using the average lottery from $f$, we can partition $S$ into two sets $\bar{S}_{\pi}(f)$ and $\underline{S}_{\pi}(f)$ as follows

$$
\bar{S}_{\pi}(f):=\left\{s^{\prime} \in S: f\left(s^{\prime}\right) \succsim f_{\pi}\right\},
$$

and $\underline{S}_{\pi}(f):=S \backslash \bar{S}_{\pi}(f)$. In other words, we partition the state set $S$ into a set of "desirable" states $\bar{S}_{\pi}(f)$, i.e., states that, under act $f$, have an associated objective lottery that the decision maker would prefer, or remain indifferent, to the objective lottery obtained by averaging with respect to $\pi$ all of the objective lotteries defined by the act; and "undesirable" states $\underline{S}_{\pi}(f)$, i.e., the remaining states in $S$.

Define

$$
\begin{aligned}
& \bar{a}_{\pi}(f):=\sum_{s \in \bar{S}_{\pi}(f)} \pi_{s}, \\
& \underline{a}_{\pi}(f):=1-\bar{a}_{\pi}(f) .
\end{aligned}
$$


The probability $\bar{a}_{\pi}(f)$ corresponds to the likelihood of choosing an arbitrary desirable state according to distribution $\pi$ and subjective lottery $f$; and $\underline{a}_{\pi}(f)$ is the complementary probability of $\bar{a}_{\pi}(f)$.

We further define the following objective lotteries associated with $f$ and $\pi$ :

$$
\begin{aligned}
\underline{f}_{\pi} & :=\left(1-\underline{a}_{\pi}(f)\right) \sum_{s \in \bar{S}_{\pi}(f)} \pi_{s} f(s)+\left(1+\bar{a}_{\pi}(f)\right) \sum_{s \in \underline{S}_{\pi}(f)} \pi_{s} f(s) \\
\bar{f}_{\pi} & :=\left(1+\underline{a}_{\pi}(f)\right) \sum_{s \in \bar{S}_{\pi}(f)} \pi_{s} f(s)+\left(1-\bar{a}_{\pi}(f)\right) \sum_{s \in \underline{S}_{\pi}(f)} \pi_{s} f(s) \\
f_{\rho, \pi} & :=\left(1-\rho \underline{a}_{\pi}(f)\right) \sum_{s \in \bar{S}_{\pi}(f)} \pi_{s} f(s)+\left(1+\rho \bar{a}_{\pi}(f)\right) \sum_{s \in \underline{S}_{\pi}(f)} \pi_{s} f(s)
\end{aligned}
$$

where $\rho \in[-1,1]$. Clearly, $f_{\pi}$ and $\bar{f}_{\pi}$ are obtained from $f_{\rho, \pi}$ by taking $\rho=1$ and $\rho=-1$, respectively. Notice that $U\left(f_{\pi}\right)=\mu(f, \pi)$ and $f_{\pi}=f_{0, \pi}$. Constant acts $\underline{f}_{\pi}$ and $\bar{f}_{\pi}$ correspond to risk-averse and risk-loving perturbations of $f_{\pi}$, respectively. The constant act $f_{\rho, \pi}$ corresponds to a perturbation of $f_{\pi}$ "in-between" $f_{-}$and $\bar{f}_{\pi}$ depending on the value of $\rho$. In the risk-averse perturbation $(\rho=1)$, the decision maker is averse to the risk that the true state is undesirable and places less weight on the probability of a desirable state relative to the likelihood that an undesirable state obtains. This observation holds more generally for $\rho$ in $(0,1]$. When $\rho$ is in $[-1,0)$, the perturbation works in the opposite direction.

To check that $f_{\rho, \pi} \in \Delta(X)$, notice that

$$
\begin{aligned}
\sum_{x \in X} f_{\rho, \pi}(x) & =(1-\rho \underline{a}) \sum_{x \in X} \sum_{s \in \bar{S}} \pi_{s} f_{s}(x)+(1+\rho \bar{a}) \sum_{x \in X} \sum_{s \in \underline{S}} \pi_{s} f_{s}(x) \\
& =(1-\rho \underline{a}) \sum_{s \in \bar{S}} \pi_{s}+(1+\rho \bar{a}) \sum_{s \in \underline{S}} \pi_{s} \\
& =(1-\rho \underline{a}) \bar{a}+(1+\rho \bar{a}) \underline{a}=1,
\end{aligned}
$$

where we have omitted the dependence on $f$ and $\pi$ to simplify the expressions.

Notice that if $f$ is a constant subjective lottery, that is, there is an objective lottery $p \in \Delta(X)$ such that $f(s)=p$ for all $s \in S$, then $\bar{S}_{\pi}(f)=S$ and $\underline{S}_{\pi}(f)=\emptyset$. Therefore, $\bar{a}_{\pi}(f)=1, \underline{a}_{\pi}(f)=0$, and $f_{\rho, \pi}=p$ for all $\rho$ and $\pi$.

We next state Axioms 5-7 to be used in our representation in Proposition 1. 
Axiom 5 (Certainty betweenness) For $f \in \mathcal{F}, p \in \Delta(X)$, and any $\alpha \in$ $(0,1), f \sim p$ implies $f \sim \alpha f+(1-\alpha) p \sim p$.

Axiom 6 (Substitution) For $f, g \in \mathcal{F}, f(s) \sim g(s)$ for all $s \in S$ implies $f \sim g$.

Axiom 7 (Bounded ambiguity preference) There exists $\pi \in \Delta(S), \pi>0$, such that $\bar{f}_{\pi} \succsim f \succsim \underline{f}_{\pi}$ for all $f \in \mathcal{F}$.

Axiom 5 was introduced by Grant and Polak (2013) and it is related to Gilboa and Schmeidler's certainty independence axiom (Gilboa and Schmeidler, 1989) as part of their multiple-prior model. Axiom 6 was also discussed by Grant and Polak (2013) and it is a weaker version of the standard monotonicity axiom.

Axiom 7 states that there exists at least one scenario $\pi$ under which the decision maker will always prefer a lottery $\bar{f}_{\pi}$, where desirable states receive a higher weight, over corresponding act $f$; and will always prefer act $f$ over a lottery $f_{\pi}$, where undesirable states receive a higher weight. In other words, Axiom 7 says that, given a distribution $\pi$ in $\Delta(S)$, and act $f$ in $\mathcal{F}$, the best perturbed lottery which can be derived from $f$ with respect to $\pi$ (the one with the highest probability that a desirable state obtains) is weakly preferred to $f$, which is weakly preferred to the worst perturbed lottery which can be derived from $f$ with respect to $\pi$ (the one with the lowest probability that a desirable state obtains). A simple behavioral test of Axiom 7 is developed in Section 4.

The bounded ambiguity preference axiom can also be interpreted as implying that ambiguity preference is bounded for each act. That is, for each act, there is a corresponding unambiguous lottery which is preferred to the act and there is an unambiguous lottery such that the act is preferred to that lottery. This captures the intuition that preferences switch from preferring an unambiguous lottery to preferring an ambiguous act when the unambiguous lottery becomes sufficiently unattractive. Under Axiom 7, a decision maker is thus averse to ambiguity when the objective lottery places a high weight on desirable states (and he thus thinks it unlikely that the act will do better), but prefers the ambiguous act when the objective lottery places a low weight on desirable states (and thus he thinks it likely that the act will do better).

Using Axioms 1-7, we next derive a mean-dispersion measure across states. This measure will represent the decision maker's preferences among 
subjective lotteries. To do so, we first define the mean absolute semideviation of the utility of $f \in \mathcal{F}$ across states as

$$
r(f, \pi):=\sum_{s \in S, U(f(s))<\mu(f, \pi)} \pi_{s}[\mu(f, \pi)-U(f(s))] .
$$

Notice that from the well-known result

$$
\sum_{k}\left|\beta_{k}\right|=\sum_{k, \beta_{k}>0} \beta_{k}-\sum_{k, \beta_{k}<0} \beta_{k}
$$

for any real vector $\left(\beta_{1}, \ldots, \beta_{n}\right)$, it follows that $r(f, \pi)$ can be also expressed as

$$
r(f, \pi)=\frac{1}{2} \sum_{s \in S} \pi_{s}|\mu(f, \pi)-U(f(s))| .
$$

Using this definition, we introduce the concept of a mean-dispersion representation across states.

Definition 1 A mean-dispersion representation across states is a given tuple $(u, \pi, \rho, V)$, where $u: X \rightarrow \mathbb{R}$ is a utility function; $\pi \in \Delta(S)$ is a probability vector over $S$; $\rho$ is a real number in $[-1,1]$; and preferences over subjective lotteries are given by

$$
V(f):=\mu(f, \pi)-\rho r(f, \pi)
$$

for all $f \in \mathcal{F}$.

The following lemma is very useful in establishing a mean-dispersion representation.

Lemma 1 For every $f \in \mathcal{F}, \pi \in \Delta(S)$, and $\rho \in[-1,1]$, the following identity holds:

$$
U\left(f_{\rho, \pi}\right)=\mu(f, \pi)-\rho r(f, \pi)=V(f) .
$$

Proof. See Appendix.

It also follows from the lemma that $V$ is a linear non-increasing function of $\rho \in[-1,1]$, for fixed $u$ and $\pi$. Using the lemma together with Theorem 1 , it is easy to see that

$$
\bar{f}_{\pi} \succsim f_{\rho, \pi} \succsim \underline{f}_{\pi},
$$


and in particular,

$$
\bar{f}_{\pi} \succsim f_{\pi} \succsim \underline{f}_{\pi}
$$

for all $f \in \mathcal{F}$.

Now, combining Theorem 1 and Axioms 1-7, we obtain our main result from this section:

Proposition 1 The relation $\succ$ on $\mathcal{F}$ satisfies Axioms 1 through 7 if and only if there exist $\pi \in \Delta(S)$ and $\rho \in[-1,1]$ such that $\succ$ admits a mean-dispersion representation $(u, \pi, \rho, V)$ where

$$
f \succ g \text { if and only if } V(f)>V(g) \text {, }
$$

for all $f, g \in \mathcal{F}$. The utility function $u$ is nonconstant and unique up to a positive linear transformation, and $\rho \in[-1,1]$. Moreover, if there exists $f$ such that $r(f, \pi)>0$, then $\rho$ is unique. That is, if there is another representation $\left(u^{\prime}, \pi, \rho^{\prime}, V^{\prime}\right)$ for $\pi$, then $\rho=\rho^{\prime}$.

Proof. See Appendix.

The next result establishes additional properties of the mean-dispersion representation. Properties 2 and 3 in Proposition 2 are introduced as axioms by Grant and Polak (2013) and are required for the proof of Proposition 3 below.

Proposition 2 If the relation $\succ$ on $\mathcal{F}$ admits a mean-dispersion representation $(u, \pi, \rho, V)$ with $V$ as in (7) and $\rho \in[0,1]$, then the following properties hold:

1. (Monotonicity) For every $f, g \in \mathcal{F}, f(s) \succsim g(s)$ for all $s \in S$ implies $f \succsim g$.

2. (Constant absolute uncertainty aversion) For any $f \in \mathcal{F}$, any three constant acts $p, q$ and $p^{\prime}$, and any $\alpha \in(0,1)$

$$
\alpha f+(1-\alpha) p \succsim \alpha p^{\prime}+(1-\alpha) p \Rightarrow \alpha f+(1-\alpha) q \succsim \alpha p^{\prime}+(1-\alpha) q .
$$

3. (Preference for complete hedges) For any finite set of acts $f_{1}, \ldots, f_{m}$ in $\mathcal{F}$ such that $f_{1} \sim f_{j}$ for all $j=2, \ldots, m$, and any constant act $p \in \Delta(X)$, if the convex combination $\alpha_{1} f_{1}+\cdots+\alpha_{m} f_{m}=p$, then $p \succsim f_{1}$. 
Proof. See Appendix.

Suppose that for a given mean-dispersion representation $(u, \pi, \rho, V)$ the corresponding vN-M utility function $U$ satisfies $U(\Delta X)=\mathbb{R}$. That is, the representation is unbounded. Then, this assumption and Proposition 2 imply that Proposition 6 from Grant and Polak (2013) (page 1373) applies for all $\rho \in[0,1]$, thus yielding the following result.

Proposition 3 If the relation $\succ$ on $\mathcal{F}$ admits a mean-dispersion representation $(u, \pi, \rho, V)$ with $V$ as in (7), $U(\Delta X)=\mathbb{R}$, and $\rho \in[0,1]$, then $\pi$ is unique. That is, if there is another representation $\left(u^{\prime}, \pi^{\prime}, \rho, V^{\prime}\right)$, then $\pi=\pi^{\prime}$.

Note that the uniqueness of $\pi$ would be directly implied from the axioms if Axiom 4 is strengthened to the unboundedness axiom in Kopylov (2007) and the critical condition in Axiom 7 is replaced by $f_{\pi} \succsim f \succsim \underline{f}_{\pi}$. In addition, it can be shown that these modifications simultaneously imply that preferences are monotone and that they satisfy Schmeidler's (1989) convexity axiom and Siniscalchi's (2009) complementary independence axiom. Thus, Corollary 3 (c),(d),(e) from Grant and Polak (2013) (page 1369) applies and the preferences also have an invariant-biseparable representation (Ghirardato et al., 2004), a vector expected utility representation (Siniscalchi, 2009), and a multiple priors representation (Gilboa and Schmeidler, 1989).

\section{A Behavioral Test of Bounded Ambiguity Preference}

In some respects, Proposition 1 is surprising since Axiom 7 may seem almost too weak. Yet in the presence of Axioms 1 through 6, which are fairly standard, we obtain a novel mean-dispersion representation. Axiom 7 does, however, involve an existential condition, which may imply difficulties in terms of observability and testability. In this section we present a behavioral example in which we can design tests that can be employed to falsify Axiom 7.

Consider the following situation: inform the decision maker that there is an urn with 100 balls, where each ball is either black or red. There are two possible states: in state $s_{1}$, there are 25 black and 75 red balls. In state $s_{2}$, there are 75 black and 25 red balls. The decision maker receives $\$ 100$ if a 
black ball is drawn and $\$ 0$ if a red ball is drawn. We denote this ambiguous act by $f$. There are two objective lotteries in $f$ :

$$
\begin{aligned}
& f\left(s_{1}\right):=(\$ 100,0.25 ; \$ 0,0.75), \\
& f\left(s_{2}\right):=(\$ 100,0.75 ; \$ 0,0.25) .
\end{aligned}
$$

Since there are only two outcomes, we can normalize utilities so that $u(\$ 100)=$ 1 and $u(\$ 0)=0$. Let $\pi:=\left(\pi_{1}, 1-\pi_{1}\right)$, with $0<\pi_{1}<1$, be a probability distribution across states. The mean-dispersion value of the risk-loving perturbed lottery derived from act $f$ is given by:

$$
V\left(\bar{f}_{\pi}\right)=0.75-0.50 \pi_{1}^{2} .
$$

Similarly, the mean-dispersion value of the risk-averse perturbed lottery derived from act $f$ is given by:

$$
V\left(\underline{f}_{\pi}\right)=0.75-\pi_{1}+0.50 \pi_{1}^{2} .
$$

Axiom 7 implies that there is some $\hat{\pi}_{1}$ such that $\bar{f}_{\hat{\pi}} \succsim f \succsim \underline{f}_{\hat{\pi}}$. Let $v:=V(f)$, then we have $V\left(\bar{f}_{\hat{\pi}}\right) \geq v \geq V\left(\underline{f}_{\hat{\pi}}\right)$. This implies that we should have $1 / 4 \leq v \leq 3 / 4$. Moreover, using (11) and (12), we obtain

$$
\sqrt{\frac{3}{2}-2 v} \geq \hat{\pi}_{1} \geq 1-\sqrt{2 v-\frac{1}{2}}
$$

Next, consider an act $g$ defined as:

$$
\begin{aligned}
& g\left(s_{1}\right):=(\$ 100,0.75 ; \$ 0,0.25), \\
& g\left(s_{2}\right):=(\$ 100,0.25 ; \$ 0,0.75) .
\end{aligned}
$$

Thus, $g$ is equivalent to $f$ except that the assignment of lotteries to states has been reversed. Like with $f$, we must have $\bar{g}_{\hat{\pi}} \succsim g \succsim \underline{g}_{\hat{\pi}}$, which yields $1 / 4 \leq \hat{v} \leq 3 / 4$ and

$$
\sqrt{2 \hat{v}-\frac{1}{2}} \geq \hat{\pi}_{1} \geq 1-\sqrt{\frac{3}{2}-2 \hat{v}}
$$

where $\hat{v}:=V(g)$. From (13) and (14), it follows that

$$
\sqrt{2 \hat{v}-\frac{1}{2}}+\sqrt{2 v-\frac{1}{2}} \geq 1
$$


and

$$
\sqrt{\frac{3}{2}-2 v}+\sqrt{\frac{3}{2}-2 \hat{v}} \geq 1 .
$$

Conditions (15) and (16) provide tests for Axiom 7. First, note that we can determine $v$ and $\hat{v}$ by using standard methods. For instance, if $h_{1}$ denotes the act that yields with certainty $\$ 100$ regardless of the state and $h_{0}$ denotes the act that yields with certainty $\$ 0$ regardless of the state, then we can use binary search (see Luce and Raiffa, 1989) to behaviorally find $\alpha_{f} \in[0,1]$ such that $f \sim \alpha_{f} h_{1}+\left(1-\alpha_{f}\right) h_{0}$. Similarly, we can find $\alpha_{g} \in[0,1]$ such that $g \sim \alpha_{g} h_{1}+\left(1-\alpha_{g}\right) h_{0}$. It follows ${ }^{2}$ that $v=\alpha_{f}$ and $\hat{v}=\alpha_{g}$. Notice that if either inequality (15) or (16) does not hold, then Axiom 7 cannot be true. This could happen when both $v$ and $\hat{v}$ are less than $3 / 8$ or both $v$ and $\hat{v}$ are greater than 5/8; in the first case inequality (15) will not hold and in the second case inequality (16) will not hold. Of course, if both inequalities hold, then we cannot falsify Axiom 7 in this case, but other acts can be added as to bound even further the range for $\hat{\pi}_{1}$ and determine if the additional inequalities are consistent or not. Also, values other than 0.25 and 0.75 can be used in defining the probabilities of the acts $f$ and $g$.

Finally, note that this approach can be used constructively to elicit $\hat{\pi}_{1}$ (if it exists) for this example. For instance, if $\alpha_{f}$ and $\alpha_{g}$ turn out to be equal to $1 / 2$ and Axioms 1-7 hold, then $\hat{\pi}_{1}$ must be in the interval $[1-\sqrt{1 / 2}, \sqrt{1 / 2}]$ (i.e., roughly between 0.293 and 0.707 ). If $\alpha_{f}=\alpha_{g}=0.4$, then $\hat{\pi}_{1}$ must be roughly in the interval $[0.452,0.548]$.

\section{The Ellsberg Paradox}

We include a formal demonstration that representation (10) can resolve Ellsberg's (1961) paradox. A similar formal demonstration in the setup involving second order acts is provided in Schneider and Nunez (2015) for Ellsberg's two-color paradox. We apply the mean-dispersion model in the AnscombeAumann framework developed here to resolve Ellsberg's three-color paradox. Consider Ellsberg's three-color paradox in which one chooses between an objective and a subjective lottery. The agent is told that an urn contains 30 red balls and 60 other balls which are either black or yellow, although the

\footnotetext{
${ }^{2}$ If either $\alpha_{f}$ or $\alpha_{g}$ does not exist, then the continuity axiom (Axiom 2) does not hold independently of whether Axiom 7 holds, but this is a separate issue.
} 
exact proportion is unknown. The agent is then provided with the following pair of binary choices:

1. Choose between (a) and (b):

(a) Receive $\$ 100$ if a red ball is drawn.

(b) Receive $\$ 100$ if a black ball is drawn.

2. Choose between (a) and (b):

(a) Receive $\$ 100$ if a red or yellow ball is drawn.

(b) Receive $\$ 100$ if a black or yellow ball is drawn.

Ellsberg (1961) observed that many people would strictly prefer 1(a) over 1(b), and 2(b) over 2(a), indicating a strict preference for betting on an outcome with a known probability over an outcome with unknown probability. Such ambiguity-averse preferences violate the axioms of subjective expected utility theory. However, such behavior is naturally accommodated by representation (10).

To illustrate, we first normalize the utility function such that $u(\$ 100)=1$ and $u(\$ 0)=0$. The state space is $S=\{0, \ldots, 60\}$, where $s \in S$ represents the number of black balls in the urn. Note that $s \in S$ fully characterizes the state of the experiment, since the number of yellow balls is $60-s$. We associate acts $f_{1}, f_{2}, f_{3}$, and $f_{4}$ with bets $1(\mathrm{a}), 1(\mathrm{~b}), 2(\mathrm{a})$, and $2(\mathrm{~b})$, respectively, as follows:

$$
\begin{aligned}
& f_{1}(s):=(1: 1 / 3 ; 0: 2 / 3), \\
& f_{2}(s):=(1: s / 90 ; 0: 1-s / 90), \\
& f_{3}(s):=(1: 1-s / 90 ; 0: s / 90), \\
& f_{4}(s):=(1: 2 / 3 ; 0: 1 / 3),
\end{aligned}
$$

for all $s \in S$, where the notation $\left(u_{1}: p_{1} ; u_{2}: p_{2}\right)$ represents the utility $u_{1}$ on a winning outcome with probability $p_{1}$ and the utility $u_{2}$ on a losing outcome with probability $p_{2}$ for each of the four acts.

For $\pi \in \Delta(S)$ define

$$
A_{\pi}:=\sum_{s=0}^{60} s \pi_{s}, B_{\pi}:=\sum_{s=0}^{60}\left|A_{\pi}-s\right| \pi_{s} .
$$


It is easy to see that $\mu\left(f_{1}, \pi\right)=1 / 3, \mu\left(f_{2}, \pi\right)=A_{\pi} / 90, \mu\left(f_{3}, \pi\right)=1-A_{\pi} / 90$, and $\mu\left(f_{4}, \pi\right)=2 / 3$. Hence, if a person has preferences $f_{1} \succ f_{2}$ and $f_{4} \succ f_{3}$, and a subjective expected utility representation holds for a given $\pi$, we must simultaneously have $\mu\left(f_{1}, \pi\right)>\mu\left(f_{2}, \pi\right)$ and $\mu\left(f_{4}, \pi\right)>\mu\left(f_{3}, \pi\right)$, which lead to the contradictory inequalities $A_{\pi}<30$ and $A_{\pi}>30$, respectively.

On the other hand, we can see how Ellsberg's paradox could be resolved by the model in Proposition 1 by noticing that $r\left(f_{1}, \pi\right)=0, r\left(f_{2}, \pi\right)=B_{\pi} / 180$, $r\left(f_{3}, \pi\right)=B_{\pi} / 180$, and $r\left(f_{4}, \pi\right)=0$. Therefore, $V\left(f_{1}, \pi\right)>V\left(f_{2}, \pi\right)$ and $V\left(f_{4}, \pi\right)>V\left(f_{3}, \pi\right)$ will hold for all $\pi$ in the set

$$
\left\{\pi \in \Delta(S): \rho \frac{B_{\pi}}{2}>\left|A_{\pi}-30\right|, \pi>0\right\} .
$$

For example, if the decision maker is consistent with Axioms 1-7, and $\pi$ in Axiom 7 corresponds to the uniform distribution $\pi_{s}=1 / 61$ for all $s$ or to a distribution close to the uniform distribution, then $\pi$ would be in set (17) and the paradox resolved under representation (10) for any $\rho$ in $(0,1]$. An analogous argument confirms that Ellsberg's two-color paradox is also resolved under similar conditions (for any $\pi$ close to the uniform distribution and any $\rho$ in $(0,1])$.

\section{Conclusion}

In this note, we have provided a simple axiomatic approach to mean-dispersion preferences in the Anscombe-Aumann (1963) framework in which the dispersion function, the utility function, the tradeoff coefficient $\rho \in[-1,1]$, and the probability distribution $\pi \in \Delta(S)$ are uniquely determined from the axioms. We subsequently showed that the model explains ambiguity aversion in Ellsberg's paradox. We also introduced a behavioral test for our novel axiom of bounded ambiguity preference. The model presented here complements the work on mean-dispersion preferences by Grant and Polak (2013), as well as the class of variational preferences (Maccheroni et al., 2006a,b) and vector expected utility preferences (Siniscalchi, 2009). While these latter representations are more general, the model presented here provides a behavioral foundation for mean-dispersion preferences with a specific form for the dispersion function which has been largely absent from the literature. 


\section{Appendix: Proofs of Results}

Proof of Lemma 1: Let $f \in \mathcal{F}, \pi \in \Delta(S)$, and $\rho \in[-1,1]$. Then

$$
\begin{aligned}
U\left(f_{\rho, \pi}\right) & =\sum_{x \in X} f_{\rho, \pi}(x) u(x) \\
& =(1-\rho \underline{a}) \sum_{x \in X} \sum_{s \in \bar{S}} \pi_{s} f_{s}(x) u(x)+(1+\rho \bar{a}) \sum_{x \in X} \sum_{s \in \underline{S}} \pi_{s} f_{s}(x) u(x) \\
& =\mu(f, \pi)-\rho\left(\underline{a} \mu(f, \pi)-\sum_{s \in \underline{S}} \sum_{x \in X} \pi_{s} f_{s}(x) u(x)\right) \\
& =\mu(f, \pi)-\rho \sum_{s \in \underline{S}} \pi_{s}[\mu(f, \pi)-U(f(s))],
\end{aligned}
$$

where for simplicity we have omitted the dependence of $\underline{a}, \bar{a}, \underline{S}$ and $\bar{S}$ on $f$ and $\pi$. Observe that $s^{\prime} \in \underline{S}_{\pi}(f)$ if and only if $f\left(s^{\prime}\right) \prec f_{\pi}$, that is, if and only if $\sum_{x \in X} f_{s^{\prime}}(x) u(x)<\mu(f, \pi)$. Hence, from (6), we get

$$
\sum_{s \in \underline{S}} \pi_{s}[\mu(f, \pi)-U(f(s))]=r(f, \pi),
$$

and therefore, $U\left(f_{\rho, \pi}\right)=\mu(f, \pi)-\rho r(f, \pi)$.

Proof of Proposition 1: We first prove that Axioms 1 through 7 are sufficient, so let us assume that those axioms hold. From Theorem 1, there exists a nonconstant affine function $u: X \rightarrow \mathbb{R}$ such that $p \succ q$ if and only if $U(p)>$ $U(q)$ for all $p, q \in \Delta(X)$. From Axiom 7, there exists $\pi \in \Delta(S)$ such that $\bar{f}_{\pi} \succsim f \succsim \underline{f}_{\pi}$ for all $f \in \mathcal{F}$. Hence, from Axiom 2, for each $f \in \mathcal{F}$ there exists $\alpha \in[0,1]$ such that $f \sim \alpha \underline{f}_{\pi}+(1-\alpha) \bar{f}_{\pi}$. Since $\alpha \underline{f}_{\pi}+(1-\alpha) \bar{f}_{\pi} \in \Delta(X)$, we can apply Lemma 1 to obtain $U\left(\alpha \underline{f}_{\pi}+(1-\alpha) \bar{f}_{\pi}\right)=\mu(f, \pi)-(2 \alpha-1) r(f, \pi)$. Now, if we set $\rho:=2 \alpha-1$, then applying Lemma 1 again we obtain $U\left(\alpha \underline{f}_{\pi}+(1-\alpha) \bar{f}_{\pi}\right)=U\left(f_{\rho, \pi}\right)$, and from Theorem 1 we conclude $f \sim$ $\alpha \underline{f}_{\pi}+(1-\alpha) \bar{f}_{\pi} \sim f_{\rho, \pi}$ with $\rho \in[-1,1]$. Thus, for each $f \in \mathcal{F}$ there exists $\rho \in[-1,1]$ such that $f \sim f_{\rho, \pi}$.

Next, we show that the following result holds:

$$
r(f, \pi)>0 \text { and } f_{\rho, \pi} \sim f_{\hat{\rho}, \pi} \Rightarrow \rho=\hat{\rho} ;
$$


for all $f \in \mathcal{F}, p \in \Delta(X)$, and $\alpha \in[0,1]$. Indeed, $f_{\rho, \pi} \sim f_{\hat{\rho}, \pi}$ implies $U\left(f_{\rho, \pi}\right)=$ $U\left(f_{\hat{\rho}, \pi}\right)$, which by Lemma 1 implies $(\rho-\hat{\rho}) r(f, \pi)=0$. Hence, $r(f, \pi)>0$ implies $\rho=\hat{\rho}$ and (18) follows.

In particular, result (18) implies that for each $f \in \mathcal{F}$, with $r(f, \pi)>0$, there is a unique $\rho \in[-1,1]$ such that $f \sim f_{\rho, \pi}$. Moreover, if we define $f^{(\alpha)}:=\alpha f+(1-\alpha) f_{\rho, \pi}$ for $\alpha \in(0,1]$, it follows from Axiom 5 that $f^{(\alpha)} \sim$ $f_{\rho, \pi}$. It is easy to see that $r\left(f^{(\alpha)}, \pi\right)=\alpha r(f, \pi)$, and so, $r(f, \pi)>0$ implies $r\left(f^{(\alpha)}, \pi\right)>0$ for all $\alpha \in(0,1]$. It is also easy to show that $f_{\rho, \pi} \sim\left(f^{(\alpha)}\right)_{\rho, \pi}$. Thus, $r(f, \pi)>0$ implies that there is a unique $\rho \in[-1,1]$ such that $f^{(\alpha)} \sim$ $\left(f^{(\alpha)}\right)_{\rho, \pi}$ for all $\alpha \in[0,1]$.

Based on the results from the previous paragraph, given $f \in \mathcal{F}$ with $r(f, \pi)>0$, observe that the function $v: \mathbb{R}^{2} \rightarrow \mathbb{R}$ defined as $v(x, y)=$ $y-\rho x$ always takes value $U\left(f_{\rho, \pi}\right)$ on the line segment $L_{\rho}(f)$ joining the points $(r(f, \pi), \mu(f, \pi))$ and $\left(0, U\left(f_{\rho, \pi}\right)\right)$. This follows from observing that for each point $(x, y)$ in $L_{\rho}(f)$, that is, a point of the form $(x, y)=\alpha(r(f, \pi), \mu(f, \pi))+$ $(1-\alpha)\left(0, U\left(f_{\rho, \pi}\right)\right)$, there corresponds act $f^{(\alpha)}:=\alpha f+(1-\alpha) f_{\rho, \pi}$ such that $v(x, y)=U\left(\left(f^{(\alpha)}\right)_{\rho, \pi}\right)=U\left(f_{\rho, \pi}\right)$. Moreover, let $f^{\prime}:=\beta f+(1-\beta) p$ with $p \in \Delta(X)$ and fixed $\beta \in(0,1]$. The line segment $L_{\rho}\left(f^{\prime}\right)$ joining points $\left(r\left(f^{\prime}, \pi\right), \mu\left(f^{\prime}, \pi\right)\right)$ and $\left(0, U\left(f_{\rho, \pi}^{\prime}\right)\right)$ is parallel to $L_{\rho}(f)$ because it has slope

$$
\frac{\mu\left(f^{\prime}, \pi\right)-U\left(f_{\rho, \pi}^{\prime}\right)}{r\left(f^{\prime}, \pi\right)}=\rho=\frac{\mu(f, \pi)-U\left(f_{\rho, \pi}\right)}{r(f, \pi)},
$$

where the right-hand side expression is the slope of $L_{\rho}(f)$. Hence, $L_{\rho}\left(f^{\prime}\right)$ is just a geometric translation of $L_{\rho}(f)$. We also have $v$ constant and equal to $U\left(f_{\rho, \pi}^{\prime}\right)=\beta U\left(f_{\rho, \pi}\right)+(1-\beta) U(p)$ on $L_{\rho}\left(f^{\prime}\right)$.

Now, let $f, g \in \mathcal{F}$ with $f \not g, r(f, \pi)>0$, and $r(g, \pi)>0$. There exist $\rho_{0}, \rho_{1} \in[-1,1]$ such that $f \sim f_{\rho_{0}, \pi}$ and $g \sim g_{\rho_{1}, \pi}$. Thus, $f_{\rho_{0}, \pi} \nsim g_{\rho_{1}, \pi}$ and $U\left(f_{\rho_{0}, \pi}\right) \neq U\left(g_{\rho_{1}, \pi}\right)$. Define $f^{(\alpha)}:=\alpha f+(1-\alpha) g_{\rho_{1}, \pi}$ and $g^{(\beta)}:=\beta g+$ $(1-\beta) f_{\rho_{0}, \pi}$ for $\alpha, \beta \in[0,1]$. As indicated above, $L_{\rho_{0}}\left(f^{(\alpha)}\right)$ and $L_{\rho_{1}}\left(g^{(\beta)}\right)$ are geometric translations (same slope) of the respective line segments $L_{\rho_{0}}(f)$ and $L_{\rho_{1}}(g)$. Also, notice that for $\alpha=\beta=1 / 2$, the corresponding line segments $L_{\rho_{0}}\left(f^{(1 / 2)}\right)$ and $L_{\rho_{1}}\left(g^{(1 / 2)}\right)$ meet at point $\left(0,\left(U\left(f_{\rho_{0}, \pi}\right)+U\left(g_{\rho_{1}, \pi}\right)\right) / 2\right)$. If the two line segments $L_{\rho_{0}}\left(f^{(1 / 2)}\right)$ and $L_{\rho_{1}}\left(g^{(1 / 2)}\right)$ are not parallel, then by continuity of the translation of line segments, there exist an infinite number of pairs $(\alpha, \beta), \alpha \neq \beta$, within a small neighborhood of $(1 / 2,1 / 2)$ in $[0,1] \times[0,1]$ such that the line segments $L_{\rho_{0}}\left(f^{(\alpha)}\right)$ and $L_{\rho_{1}}\left(g^{(\beta)}\right)$ intersect at a unique point. Choosing any pair $(\alpha, \beta)$ such that $\alpha+\beta \neq 1$ in that neighborhood, the 
respective intersection point corresponds to an act $h$ such that $h \sim f^{(\alpha)}$ and $h \sim g^{(\beta)}$, from which we obtain $f^{(\alpha)} \sim g^{(\beta)}$ and then, $\left(f^{(\alpha)}\right)_{\rho_{0}, \pi} \sim\left(g^{(\beta)}\right)_{\rho_{1}, \pi}$ and $U\left(\left(f^{(\alpha)}\right)_{\rho_{0}, \pi}\right)=U\left(\left(g^{(\beta)}\right)_{\rho_{1}, \pi}\right)$. But this together with $\alpha+\beta \neq 1$ imply $U\left(f_{\rho_{0}, \pi}\right)=U\left(g_{\rho_{1}, \pi}\right)$, a contradiction. Thus, the line segments $L_{\rho_{0}}\left(f^{(1 / 2)}\right)$ and $L_{\rho_{1}}\left(g^{(1 / 2)}\right)$ are parallel, and so, $L_{\rho_{0}}(f)$ and $L_{\rho_{1}}(g)$ are also parallel. Therefore, we must have $\rho_{0}=\rho_{1}$.

Summarizing, there exists a unique $\rho \in[-1,1]$ such that $f \sim f_{\rho, \pi}$ for all $f \in \mathcal{F}$ such that $r(f, \pi)>0$. On the other hand, since $\pi>0$, if $r(f, \pi)=0$, then we have $U(f(s))=\mu(f, \pi)=U\left(f_{\pi}\right)$ for all $s \in S$. Hence, $f(s) \sim f_{\pi}$ for all $s \in S$ and by Axiom 6, we obtain $f \sim f_{\pi}$. Therefore, $f \sim f_{\pi}$ if and only if $r(f, \pi)=0$. It follows that if we define $V$ as

$$
V(f):= \begin{cases}U\left(f_{\rho, \pi}\right) & \text { if } r(f, \pi)>0, \\ U\left(f_{\pi}\right) & \text { if } r(f, \pi)=0,\end{cases}
$$

then $V$ is well-defined and $V(f)=\mu(f, \pi)-\rho r(f, \pi)$ for all $f$. Moreover, $f \succ g$ if and only if exactly one of the following statements holds true

$$
\begin{aligned}
& f_{\rho, \pi} \succ g_{\rho, \pi} \text { for } r(f, \pi)>0 \text { and } r(g, \pi)>0 \text {, } \\
& f_{\rho, \pi} \succ g_{\pi} \text { for } r(f, \pi)>0 \text { and } r(g, \pi)=0 \text {, } \\
& f_{\pi} \succ g_{\rho, \pi} \text { for } r(f, \pi)=0 \text { and } r(g, \pi)>0 \text {, } \\
& f_{\pi} \succ g_{\pi} \text { for } r(f, \pi)=0 \text { and } r(g, \pi)=0 \text {, }
\end{aligned}
$$

if and only if $V(f)>V(g)$.

In the second part of the proof, we need to show that the axioms are necessary. However, Axioms 1 through 4 are common in the literature and the proof that they are necessary is standard, so that we omit it. Hence, we only concentrate on proving the necessity of the non-traditional Axioms 5, 6 , and 7 .

Notice that $f \sim p$ implies $V(f)=U(p)$. On the other hand, $V(\alpha f+(1-$ $\alpha) p)=\alpha V(f)+(1-\alpha) U(p)=V(f)=U(p)$. Hence, $\alpha f+(1-\alpha) p \sim f \sim p$ and Axiom 5 follows. Next, notice that if $f(s) \sim g(s)$ for all $s \in S$, then $U(f(s))=U(g(s))$ for all $s$. It follows that $\mu(f, \pi)=\mu(g, \pi)$ and $r(f, \pi)=$ $r(g, \pi)$. Hence, $V(f)=V(g)$ and so, $f \sim g$ and Axiom 6 follows. Finally, for any act $f$ we have $V(f)=U\left(f_{\rho, \pi}\right)=V\left(f_{\rho, \pi}\right)$, which implies $f \sim f_{\rho, \pi}$. On the other hand, as indicated in (8), we clearly have $\bar{f}_{\pi} \succsim f_{\rho, \pi} \succsim \underline{f}_{\pi}$, from which we obtain $\bar{f}_{\pi} \succsim f \succsim \underline{f}_{\pi}$, and the statement of Axiom 7 also holds. 
For the rest of this section we denote by $e$ the all-ones vector and by $e_{k}$ the $k$-th canonical vector in $\mathbb{R}^{n}$. We denote by $\delta_{i j}$ the Kronecker delta function, that is, $\delta_{i j}=0$ if $i \neq j$ and $\delta_{i j}=1$ if $i=j$ for all $i, j \in\{1, \ldots, n\}$.

Lemma 2 Given $\rho \in[0,1]$ and a vector $\beta \in \mathbb{R}^{n}$ such that $\beta \geq 0$ and $\beta^{T} e=1$, let $v: \mathbb{R}^{n} \rightarrow \mathbb{R}$ be the following real-valued function:

$$
v(x):=\beta^{T} x-\frac{\rho}{2} \sum_{i=1}^{n} \beta_{i}\left|\beta^{T} x-x_{i}\right| .
$$

Then, $v$ is monotone. That is,

$$
x \leq y \text { implies } v(x) \leq v(y),
$$

for all $x, y$ in $\mathbb{R}^{n}$.

Proof. First, given any $x \in \mathbb{R}^{n}, \lambda \geq 0$, and $k \in\{1, \ldots, n\}$, we prove that $v\left(x+\lambda e_{k}\right) \geq v(x)$. Notice that the result is obviously true for $\lambda=0$, so that we assume $\lambda>0$. Consider the following sets of indexes

$$
\begin{aligned}
& A:=\left\{j: \beta^{T} x>x_{j}\right\}, \\
& B:=\left\{j: \beta^{T} x+\lambda \beta_{k}>x_{j}+\lambda \delta_{j k}\right\} .
\end{aligned}
$$

It is easy to show that $A \backslash\{k\} \subset B$ and if $k \notin A$ then $k \notin B$. Hence,

$$
\begin{aligned}
v\left(x+\lambda e_{k}\right) & =\beta^{T}\left(x+\lambda e_{k}\right)-\frac{\rho}{2} \sum_{i=1}^{n} \beta_{i}\left|\beta^{T}\left(x+\lambda e_{k}\right)-\left(x_{i}+\lambda \delta_{i k}\right)\right| \\
& =\beta^{T} x+\lambda \beta_{k}-\rho \sum_{i \in B} \beta_{i}\left(\beta^{T} x-x_{i}+\lambda\left(\beta_{k}-\delta_{i k}\right)\right) \\
& =\beta^{T} x+\lambda \beta_{k}\left(1-\rho \sum_{i \in B} \beta_{i}\right)-\rho \sum_{i \in B} \beta_{i}\left(\beta^{T} x-x_{i}-\lambda \delta_{i k}\right)
\end{aligned}
$$

If $k \notin A$, then

$$
v\left(x+\lambda e_{k}\right)=v(x)+\lambda \beta_{k}\left(1-\rho \sum_{i \in B} \beta_{i}\right)-\rho \sum_{i \in B \backslash A} \beta_{i}\left(\beta^{T} x-x_{i}\right) \geq v(x) .
$$


If $k \in A$ and $k \in B$, then

$$
v\left(x+\lambda e_{k}\right)=v(x)+\rho \lambda \beta_{k}+\lambda \beta_{k}\left(1-\rho \sum_{i \in B} \beta_{i}\right)-\rho \sum_{i \in B \backslash A} \beta_{i}\left(\beta^{T} x-x_{i}\right) \geq v(x) .
$$

If $k \in A$ and $k \notin B$, then

$$
v\left(x+\lambda e_{k}\right)=v(x)+\rho \beta_{k}\left(\beta^{T} x-x_{k}\right)+\lambda \beta_{k}\left(1-\rho \sum_{i \in B} \beta_{i}\right)-\rho \sum_{i \in B \backslash A} \beta_{i}\left(\beta^{T} x-x_{i}\right) \geq v(x) .
$$

Therefore, we always get $v\left(x+\lambda e_{k}\right) \geq v(x)$ in all cases. To conclude the proof, notice that when $x \leq y$ we can write

$$
y=\frac{1}{n} \sum_{k=1}^{n}\left(x+\lambda_{k} e_{k}\right)
$$

where $\lambda_{k}:=n\left(y_{k}-x_{k}\right) \geq 0$ for all $k$. From the previous result, we know that $v\left(x+\lambda_{k} e_{k}\right) \geq v(x)$ for all $k$. Hence, since $v$ is a concave function, we obtain

$$
v(x) \leq \frac{1}{n} \sum_{k=1}^{n} v\left(x+\lambda_{k} e_{k}\right) \leq v\left(\frac{1}{n} \sum_{k=1}^{n}\left(x+\lambda_{k} e_{k}\right)\right)=v(y)
$$

and the result follows.

Proof of Proposition 2: Property 1 follows from Lemma 2 by taking $n:=|S|, x_{s}:=U(f(s))$ and $y_{s}:=U(g(s))$ for all $s \in S$, and $\beta:=\pi$. Since $f(s) \succsim g(s)$ for all $s$, we obtain $x \geq y$, which implies $V(f)=v(x) \geq v(y)=$ $V(g)$, and so $f \succsim g$. Property 2 follows by noticing that $\alpha f+(1-\alpha) p \succsim$ $\alpha p^{\prime}+(1-\alpha) p$ implies $\alpha V(f)+(1-\alpha) U(p) \geq \alpha U\left(p^{\prime}\right)+(1-\alpha) U(p)$, which implies $\alpha V(f)+(1-\alpha) U(q) \geq \alpha U\left(p^{\prime}\right)+(1-\alpha) U(q)$, which yields $\alpha f+(1-$ $\alpha) q \succsim \alpha p^{\prime}+(1-\alpha) q$. Finally, Property 3 follows from noticing that $f_{1} \sim f_{j}$ for all $j$ implies $V\left(f_{1}\right)=V\left(f_{j}\right)$ for all $j$. Moreover, $\alpha_{1} f_{1}+\cdots+\alpha_{m} f_{m}=p$ implies $V\left(\alpha_{1} f_{1}+\cdots+\alpha_{m} f_{m}\right)=U(p)$, but since $V$ is concave on $\mathcal{F}$, we have $V\left(\alpha_{1} f_{1}+\cdots+\alpha_{m} f_{m}\right) \geq \alpha_{1} V\left(f_{1}\right)+\cdots+\alpha_{m} V\left(f_{m}\right)=V\left(f_{1}\right)$. Therefore, $U(p) \geq V\left(f_{1}\right)$ and hence, $p \succsim f_{1}$.

\section{Acknowledgments}

We thank Marciano Siniscalchi for helpful comments and suggestions. The usual disclaimers apply. 


\section{References}

Al-Najjar, N.I., L.I. De Castro. 2010. Observability and second-order acts. Tech. rep., Kellogg School of Management, Northwestern University. Center for Mathematical Studies in Economics and Management Science, No. 1531.

Anscombe, F.J., R.J. Aumann. 1963. A definition of subjective probability. The Annals of Mathematical Statistics 34(1) 199-205.

Ellsberg, D. 1961. Risk, ambiguity, and the Savage axioms. The Quarterly Journal of Economics 75(4) 643-669.

Ergin, H., F. Gul. 2009. A theory of subjective compound lotteries. Journal of Economic Theory 144(3) 899-929.

Ghirardato, P., F. Maccheroni, M. Marinaccia. 2004. Differentiating ambiguity and ambiguity attitude. Journal of Economic Theory 118(2) 133-173.

Gilboa, I., D. Schmeidler. 1989. Maxmin expected utility with non-unique prior. Journal of Mathematical Economics 18(2) 141-153.

Grant, S., B. Polak. 2013. Mean-dispersion preferences and constant absolute uncertainty aversion. Journal of Economic Theory 148 1361-1398.

Grant, S., B. Polak, T. Strzalecki. 2009. Second-order expected utility. Tech. rep., Yale University.

Klibanoff, P., M. Marinacci, S. Mukerji. 2005. A smooth model of decision making under ambiguity. Econometrica 73(6) 1849-1892.

Kopylov, I. 2007. Procedural rationality in the multiple priors model. Mimeo, University of California, Irvine.

Kreps, D.M. 1988. Notes on the Theory of Choice. Westview Press, Inc., Boulder, CO.

Luce, D., H. Raiffa. 1989. Games and Decisions: Introduction and Critical Survey. Dover Publications, Inc.

Maccheroni, F., M. Marinacci, A. Rustichini. 2006a. Ambiguity aversion, robustness, and the variational representation of preferences. Econometrica 74(6) 1447-1498. 
Maccheroni, F., M. Marinacci, A. Rustichini. 2006b. Dynamic variational preferences. Journal of Economic Theory 128(1) 4-44.

Nau, R.F. 2006. Uncertainty aversion with second-order utilities and probabilities. Management Science 52(1) 136-145.

Neilson, W.S. 2010. A simplified axiomatic approach to ambiguity aversion. Journal of Risk Uncertainty 43 113-124.

Schmeidler, D. 1989. Subjective probability and expected utility without additivity. Econometrica 57(3) 571-587.

Schneider, M., M. Nunez. 2015. A simple mean-dispersion model of ambiguity attitudes. Journal of Mathematical Economics. 58(C) 25-31.

Seo, K. 2009. Ambiguity and second-order belief. Econometrica 77(5) 15751605 .

Siniscalchi, M. 2009. Vector expected utility and attitudes toward variation. Econometrica 77(3) 801-855. 